\title{
BMC Pharmacology and Toxicology reviewer acknowledgement 2015
}

Christopher Morrey

\section{Contributing reviewers}

The editors of BMC Pharmacology and Toxicology would like to thank all our reviewers who have contributed to the journal in Volume 16 (2015).

\section{Lamya Alnaim}

Saudi Arabia

Guzmán Alvarez

Uruguay

Solomon Amade

Ethiopia

Richard Amison

UK

Salvatore Amoroso

Italy

Karl-Erik Andersson

USA

Paulo Arnaldo

Mozambique

Oscar Arrieta

Mexico

Alfred Balch

USA

Douglas Ball

Philippines

Jo Barnes

New Zealand

Perihan Başhan

Turkey

Frank Beier

Canada
Raja Benkirane
Morocco

Amanda Bess

USA

Jiraganya Bhongsatiern

USA

Margherita Brindisi

Italy

Stephen Britland

UK

Simone Brogi

Italy

Florent Busi

France

Rainer W Bussmann

USA

Gillian Butler-Browne

France

Sabrina Calil-Elias

Brazil

Sarah Campbell

USA

Domenico Capone

Italy

Mariella Carrieri

Italy

\author{
Mauro Cataldi \\ Italy
}

Mauricio Chagas Da Silva

Canada

Kathryn Chapman

UK

Sara Cheleschi

Italy

Yingjia Chen

USA

Imti Choonara

UK

Lubica Cibickova

Czech Republic

Bart Crielaard

Netherlands

Elena Crisa

Italy

Anna Czlonkowska

Poland

Nissar Darmani

USA

Fabrizio De Ponti

Italy

Saskia De Wildt

Netherlands

Correspondence: christopher.morrey@biomedcentral.com

BioMed Central, Floor 6, 236 Gray's Inn Road, London WC1X 8HB, United

Kingdom 
Monorama Deb

India

Chris Delcher

USA

Domenico Delfino

Italy

John Dempster

UK

Antonello Di Paolo

Italy

Kawa Dizaye

Iraq

Matthew Drake

USA

Thomas Efferth

Germany

Damon Eisen

Australia

Omar Elmi

Malaysia

Suleiman El-Sharif

United Arab Emirates

Eric Engleman

USA

Elena Enioutina

USA

Sebnem Eren Cevik

Turkey

Yu Fang

China

Wissam Faour

Lebanon

Qiping Feng

USA

Peter Ferenci

Austria

Carmela Fimognari

Italy

Carmine Finelli

Italy

Andrea Fiorillo

Italy

Sheryl Flanagan

Canada
Samuel Fountain

UK

Dana García

USA

Stephen Gbedema

Ghana

Yubin Ge

USA

Giorgio Gentile

Italy

Saeid Ghavami

Canada

Alessandra Gianoncelli

Italy

Jean-Pierre Gillet

Belgium

Ioannis Gioulbasanis

Greece

Danijela Gnjidic

Australia

Helen Griffiths

UK

Tai Guo

USA

Manjeeta Gupta

India

Peter Hansell

Sweden

Mohamed Hassan

USA

Medardo Hernández

Spain

Mohammad Hojjat-Farsangi

Sweden

Joseph Horzempa

USA

Tzu-Bou Hsieh

Canada

Yi-Shin Huang

Taiwan

Risto Huupponen

Finland

Masahiro Ikeda

Japan
Masahide Ikeguchi

Japan

Manish Issar

USA

Shazia Jamshed

Malaysia

Tong-Rong Jan

Taiwan

Sophia Johnson

USA

Ebru Karpuzoglu

USA

Imit Kaur

USA

Ron Keizer

Sweden

Ulrike Kemmerling

Chile

Martin Kimmel

Germany

Eugene Kiyatkin

USA

Curtis Klaassen

USA

Jos Kosterink

Netherlands

Sperry Kotsianas

USA

Detlef Krieter

Germany

Laura Kubik

USA

Subramaniansenthil Kumaran

India

Chun Shing Kwok

UK

Mathew Yamoah Kyei

Ghana

Malcolm Lader

UK

Vincenzo Lariccia

Italy

Cecily Rosemary Latha

India 
John Lavelle

USA

Dario Lehoux

Canada

Shing-Jong Lin

Taiwan

Duan Liu

USA

Kai Liu

USA

Xiaoxi Liu

USA

Tingting Liu

USA

Jie Liu

USA

Vincenza Rita Lo Vasco

Italy

Diomedes Logothetis

USA

Bal Lokeshwar

USA

Yi Lu

USA

Francesca Luchetti

Italy

Simona Magi

Italy

Anke Hilse Maitland-Van Der Zee

Netherlands

Francois Marceau

Canada

Zoratti Mario

Italy

Timothy Martland

UK

Wojte Marusza

Poland

María-Victoria Mateos

Spain

Isao Matsui-Yuasa

Japan

Lynn McPherson

USA
Bruce Melancon

USA

Yongfan Men

USA

Torri Metz

Colombia

Tim Millar

UK

Seigo Minami

Japan

Tomoya Mita

Japan

Maria Miteva

France

Julio Cesar Morales-Medina

Mexico

Wondemagegn Mulu

Ethiopia

Karl Nocka

USA

Lucy Okell

UK

Olufunmiso Olusola Olajuyigbe

South Africa

Pierluigi Paggiaro

Italy

Frank Paloucek

USA

Manlio Palumbo

Italy

Hitesh Pandya

UK

Nazareno Paolocci

USA

Roberto Passera

Italy

Davide Pastorelli

Italy

Aarti Patel

New Zealand

Vidya Perera

USA

Elisabetta Poluzzi

Italy
Carol Poskay

USA

Casey Quinlan

USA

Rapur Ram

India

Rubhana Raqib

Bangladesh

Balmiki Ray

USA

Jerrie Refuerzo

USA

Carlo Reggiani

Italy

Roberto Rimondini Italy

Anka Roehr

Germany

Joseph Rower

USA

Stephen Safrany

UK

Anke Schiedel

Germany

Verena Schneider-Lindner

Germany

Yutaka Seino

Japan

Abhishek Sharma

USA

Filomena Silva

Portugal

Anurag Singh

USA

Ken Soderstrom

USA

Jean Tafforeau

Belgium

Yoshikazu Takaesu

Japan

Giovanni Tarantino

Italy

Anthony Taylor

UK 
Cheong Teng

Malaysia

Manish Tiwari

USA

Sarah Tonkin-Crine

UK

Anna Toso

Italy

Pramote Tragulpiankit

Thailand

Nam Tran

USA

Arnaud Trébucq

France

Gianluca Trifirò

Italy

Fulvio Ursini

Italy

Johannes Van Der Wouden

Netherlands
Benjamin Van Tassell

USA

Sarah Vecchi

Italy

Raja Venkatasubramanian

USA

Margreee Vissers

New Zealand

Ju Wang

USA

Jeffrey Wang

USA

Yunbiao Wang

China

Yibo Wang

Canada

Phurpa Wangchuk

Australia

Dereje Woldemichael

Ethiopia
Joella Xu

USA

Josef Yayan

Germany

Venkata Yellepeddi

USA

Frederique Yiannikouris USA

Ebru Yildirim

Turkey

Tian Yu

USA

Jiabin Zhang

USA

Weixia Zhong

USA

Meijia Zhou

USA

Michele Zoli

Italy 